\title{
CMEARTICLE
}

\section{Clinics in diagnostic imaging (154)}

Puay Joo $\underline{\text { Lim }}^{1}$, MBBS, FRCR, Sumer Nrupendra Shikhare ${ }^{1}$, MBBS, FRCR, Wilfred CG $\underline{\text { eh }}^{1}$, MBBS, FRCR

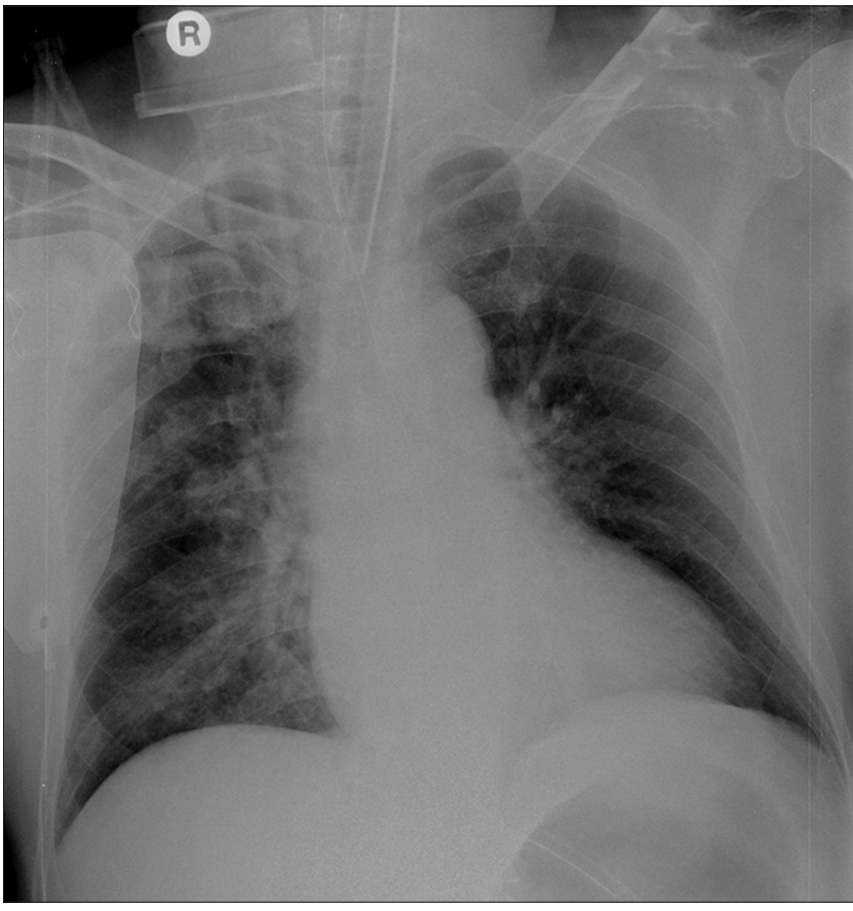

Fig. 1 Frontal chest radiograph.

\section{CLINICAL PRESENTATION}

A 59-year-old man with a notable history of major depression was found by his wife to be unconscious and foaming at the mouth. On arrival at the emergency department, the patient was unresponsive, with a Glasgow Coma Scale score of 6 . On physical examination, the patient's pupils were $3.0 \mathrm{~mm}$ wide bilaterally, with a sluggish light reflex. His blood pressure was 150/67 mmHg, heart rate was 90 beats per min, oxygen saturation was $100 \%$ on high-flow oxygen and the

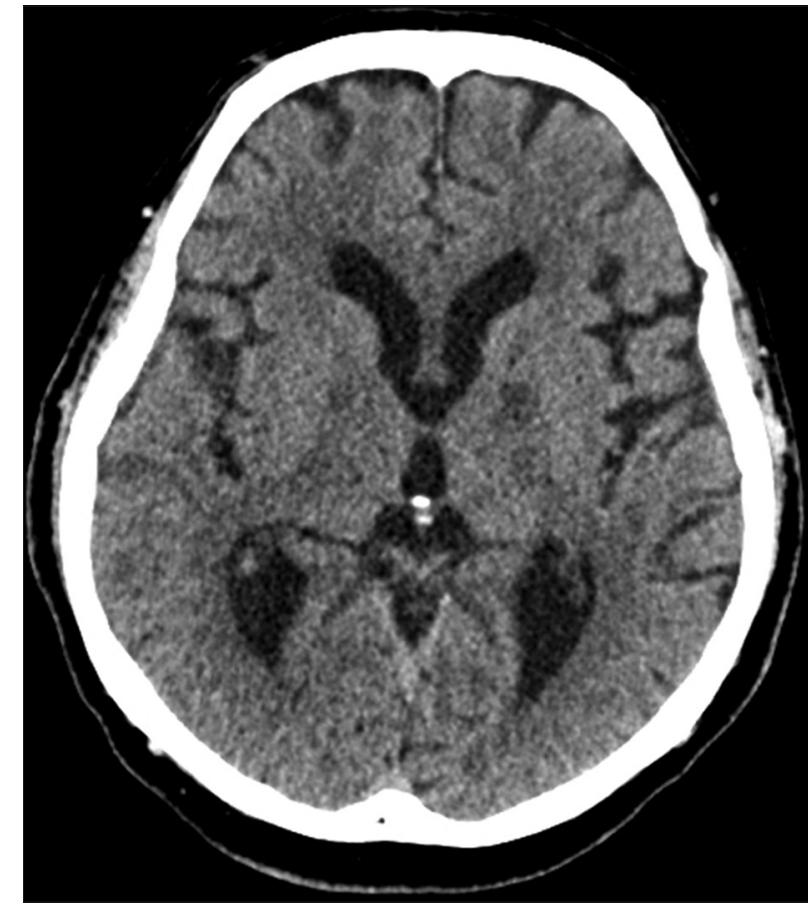

Fig. 2 Unenhanced CT image taken at the level of the basal ganglia.

pain score was 0 . Endotracheal intubation was subsequently performed for airway protection. Initial arterial blood gas analysis showed the following: $\mathrm{pH} 7.37$ (7.35-7.45); $\mathrm{pO}_{2} 260.9$ (75.0-100.0) mmHg; $\mathrm{pCO}_{2} 31.7$ (35.0-45.0) mmHg; and $\mathrm{O}_{2}$ saturation of $99.5 \%(96.0 \%-100.0 \%)$. The patient's creatinine level was 341 (59-104) $\mu \mathrm{mol} / \mathrm{L}$. A chest radiograph (Fig. 1) and unenhanced computed tomography (CT) of the brain were performed (Fig. 2). What do these images show? What is the diagnosis? 

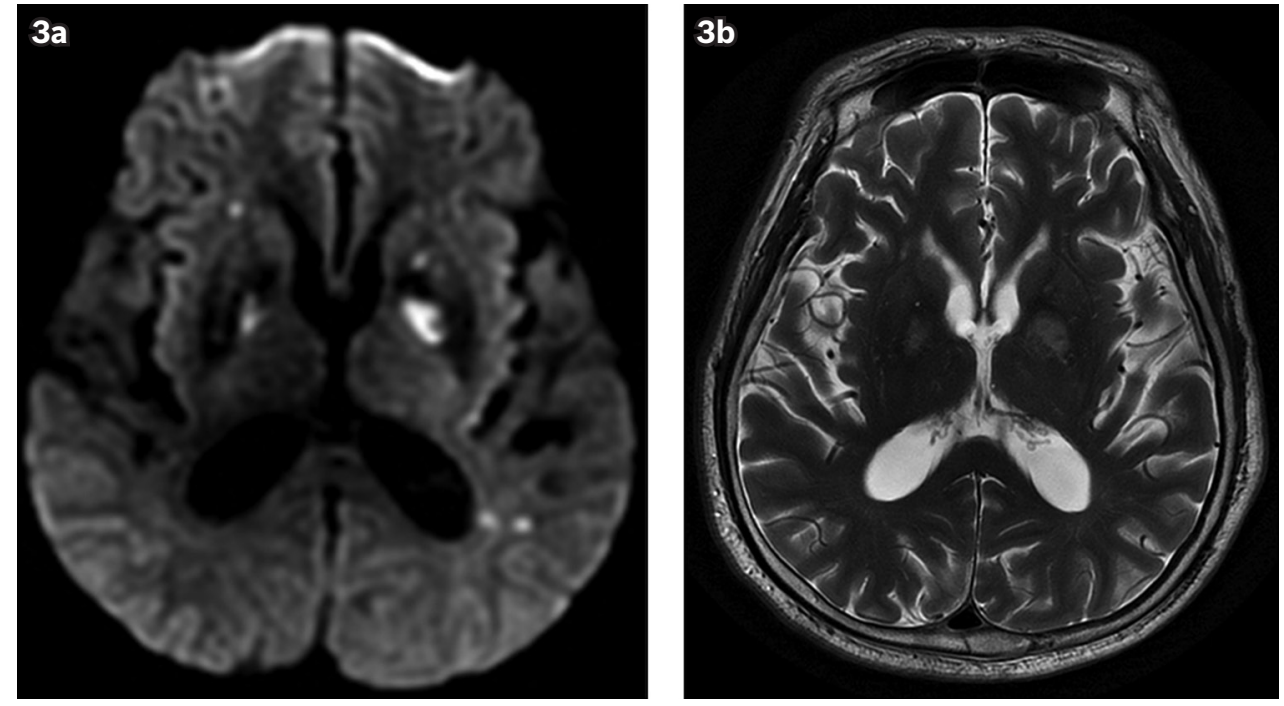

Fig. 3 (a) DW and (b) T2-W MR images show bilateral symmetrical areas of T2 prolongation and restricted diffusion in the globi pallidi. The affected sites are best seen on the DW MR image.

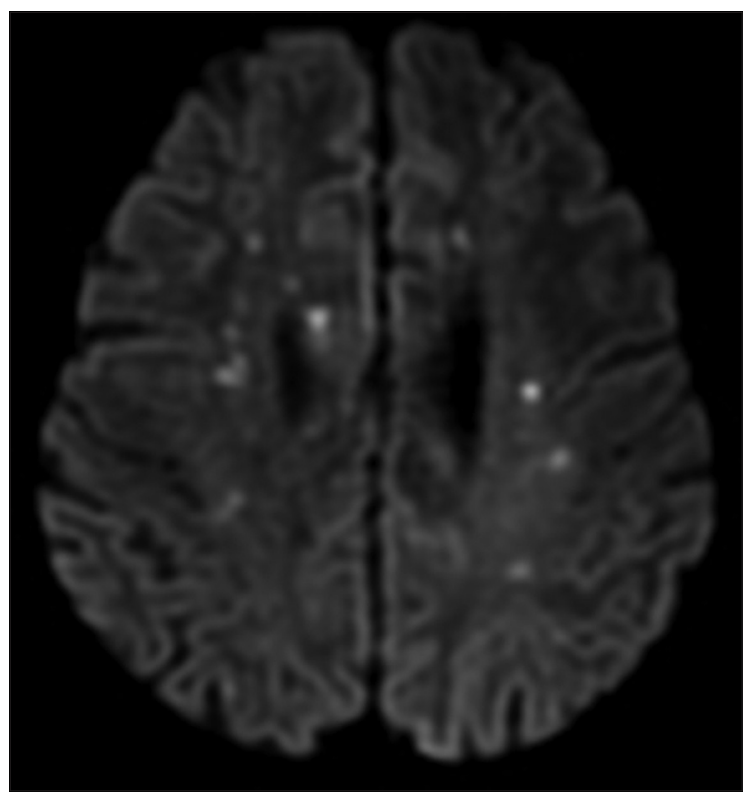

Fig. 4 DW MR image shows bilateral asymmetric areas of restricted diffusion in the white matter of both frontal and parietal lobes.

\section{IMAGE INTERPRETATION}

The chest radiograph shows bilateral perihilar air space opacities that are typical of pulmonary oedema (Fig. 1). CT images demonstrate symmetrical ill-defined areas of hypoattenuation involving the medial aspects of both lentiform nuclei (Fig. 2). No intracranial haemorrhage is evident.

\section{DIAGNOSIS}

Carbon monoxide (CO) poisoning.

\section{CLINICAL COURSE}

Further history obtained from the patient's wife confirmed that the patient had attempted suicide by ingesting multiple medications and staying in a closed room with a pot of burning charcoal. The patient's carboxyhaemoglobin level (venous sample) was tested and found to be $11.8 \%$ (> $15.0 \%$ is considered toxic). Magnetic

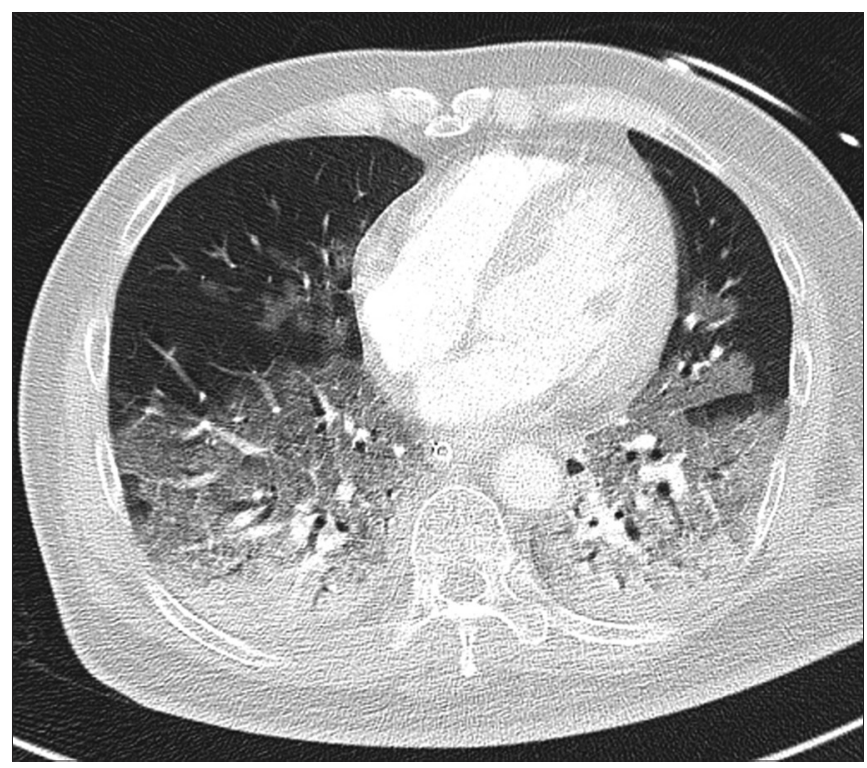

Fig. 5 CT image of the thorax shows typical areas of intense parenchymal opacification in the dependent lung and nondependent ground-glass opacification, consistent with acute respiratory distress syndrome.

resonance (MR) imaging of the brain showed symmetrical increased signal in both globi pallidi on diffusion-weighted (DW) and T2-weighted sequences (Fig. 3). Multiple small, scattered foci of restricted diffusion involving the white matter of the frontal and parietal lobes in both cerebral hemispheres (Fig. 4) were also noted.

Despite aggressive treatment, the patient's level of consciousness remained poor. His subsequent chest radiograph revealed bilateral prominent interstitial markings with bilateral basal infiltrates. Repeat arterial blood gas analysis showed the following: $\mathrm{pH} 7.420$ (7.350-7.450); $\mathrm{pO}_{2} 37.0$ (75.0-100.0) mmHg; $\mathrm{pCO}_{2} 32.0$ (35.0-45.0) mmHg; and $\mathrm{O}_{2}$ saturation $71.0 \%(96.0 \%-100.0 \%)$. In view of the declining oxygen saturations, CT pulmonary angiogram was performed (Fig. 5) to exclude underlying pulmonary embolism. CT pulmonary angiogram demonstrated symmetrical, 
dependent consolidation in both the lower lobes, with smooth septal thickening and nondependent ground-glass changes in the right upper and middle lobes, as well as the lingula segments of the left upper lobe. Findings were consistent with acute respiratory distress syndrome (ARDS) secondary to $\mathrm{CO}$ poisoning. There was no pulmonary embolism.

Subsequently, the patient became bradycardic, hypoxic and hypotensive. His pulse rate was 50 beats per minute, $\mathrm{SpO}_{2}$ was $60 \%$ and systolic blood pressure was $40 \mathrm{mmHg}$. He had progressively increasing ventilator requirements, and serial chest radiographs revealed progressively worsening changes of ARDS. The patient's condition continued to deteriorate and he eventually passed away.

\section{DISCUSSION}

Carbon monoxide is a colourless, odourless gas produced during incomplete combustion of carbon-containing compounds. $\mathrm{CO}$ inhalation is the most common cause of fatal poisoning worldwide, ${ }^{(1-3)}$ demonstrating the severest effect on the brain due to its high oxygen requirement. The pathophysiology of $\mathrm{CO}$ toxicity primarily involves cellular hypoxia due to impaired oxygen transport by haemoglobin, as CO binds to haemoglobin more avidly than oxygen (230-270 times). Other mechanisms of $\mathrm{CO}$ toxicity include direct toxic effects on respiratory cytochromes, brain lipid peroxidation, leucocyte-mediated inflammatory changes in the brain, and release of nitric oxide free radical from platelet and vascular endothelium.

Clinically, patients with mild CO poisoning may present with headache, malaise, nausea, vomiting and signs of subtle cognitive impairment such as memory deficits, impaired arithmetic skills and dyspraxia. Patients with more severe intoxication usually demonstrate neurological signs such as motor dysfunction, particularly impaired gait and balance. Less commonly, signs of sensory impairment such as cortical blindness, tinnitus and deafness may be observed. Loss of consciousness is a common presentation, although this is usually transient. Persistent loss of consciousness, even after normobaric oxygen administration, suggests severe intoxication, although concomitant consumption of other sedative drugs or alcohol can complicate the presentation. Some patients who demonstrate apparent good recovery from the acute symptoms of CO poisoning may later develop delayed neurological syndrome up to five weeks after exposure. This is characterised by neuropsychiatric and behavioural features, as well as motor dysfunction. Patients may present with mental retardation, emotional disorders, urinary and faecal incontinence, as well as motor disorders such as gait disturbances and features of Parkinsonism. CO poisoning can also result in acute pulmonary oedema, which may be secondary to cardiac ischaemia from CO-induced hypoxia or non-cardiogenic pulmonary oedema as a direct result of $\mathrm{CO}$ inhalation.

Laboratory testing for carboxyhaemoglobin levels can be performed using either arterial or venous samples, with values derived from venous samples closely matching those from arterial samples. ${ }^{(4)}$ In a nonsmoker, the average carboxyhaemoglobin level is $1 \%$, with levels of up to $15 \%$ seen in heavy smokers. ${ }^{(5)}$ The clinical presentation and outcome of patients with $\mathrm{CO}$ poisoning, however, shows poor correlation with blood carboxyhaemoglobin levels. ${ }^{(6)}$

The basal ganglia have high metabolic activity and are thus prone to systemic disease processes and toxic metabolic abnormalities. ${ }^{(7)}$ In particular, the globus pallidus is most commonly involved in $\mathrm{CO}$ poisoning. The globus pallidus may, however, be spared in some patients who demonstrate other patterns of brain injury, as described later in the Discussion. Although MR imaging is superior for evaluation of the basal ganglia, CT is often the first line of investigation, especially in the emergency setting. In acute CO poisoning, CT of the brain usually demonstrates bilateral symmetrical hypoattenuation of the globi pallidi as a result of underlying necrosis. This is seen on MR imaging as areas of increased signal on T2-weighted, fluid attenuated inversion recovery (FLAIR) and DW sequences. ${ }^{(8)}$ Contrast-enhanced T1-weighted images may also demonstrate patchy or peripheral enhancement of the necrotic globus pallidus in cases of acute poisoning.

Several other patterns of brain injury in $\mathrm{CO}$ poisoning have also been described. These include injury to other structures such as the rest of the basal ganglia, thalamus, brainstem and cerebellum, diffuse hypoxic-ischaemic encephalopathy, focal cortical injury, diffuse brain atrophy and cerebral white matter demyelination. ${ }^{(8)}$ Structures such as the caudate nucleus, putamen, thalamus, brainstem and cerebellum may also be involved in acute $\mathrm{CO}$ poisoning, albeit less commonly, demonstrating increased signal on T2-weighted and FLAIR images with an asymmetrical distribution. ${ }^{(9)}$ Involvement of these structures may also be delayed, manifesting up to five days after the acute episode of poisoning. Cerebellar and brainstem signal abnormalities are a manifestation of more severe poisoning, as the posterior structures have a higher threshold for hypoxic injury. ${ }^{(9)}$

Diffuse hypoxic-ischaemic encephalopathy usually develops only in cases of acute and severe $\mathrm{CO}$ poisoning or prolonged exposure, where autoregulatory mechanisms to increase cerebral blood flow have been overwhelmed due to exhaustion of cardiovascular homeostatic mechanisms. This usually presents as diffuse increased signal in the cortex on T2-weighted or FLAIR images. Focal cortical injury is considerably less common, usually affecting the temporal lobe or hippocampus. ${ }^{(9)}$

Diffuse brain atrophy is a late manifestation of CO poisoning resulting from neuronal cell death and apoptosis secondary to transient hypoxia and direct $\mathrm{CO}$ toxicity. ${ }^{(8)}$ This manifests on both $\mathrm{CT}$ and MR images as sulcal widening and increased ventricular size, disproportionate to patient age. Cerebral white matter demyelination is also a chronic manifestation of CO poisoning, ${ }^{(9)}$ with the affected white matter structures demonstrating decreased signal on T1-weighted MR images and increased signal on T2-weighted MR images. The most common sites of involvement are the periventricular white matter and centrum semiovale; the subcortical white matter, corpus callosum and both the internal and external capsules are involved in cases of severe poisoning. ${ }^{(10)}$ It is postulated that white matter demyelination is responsible 


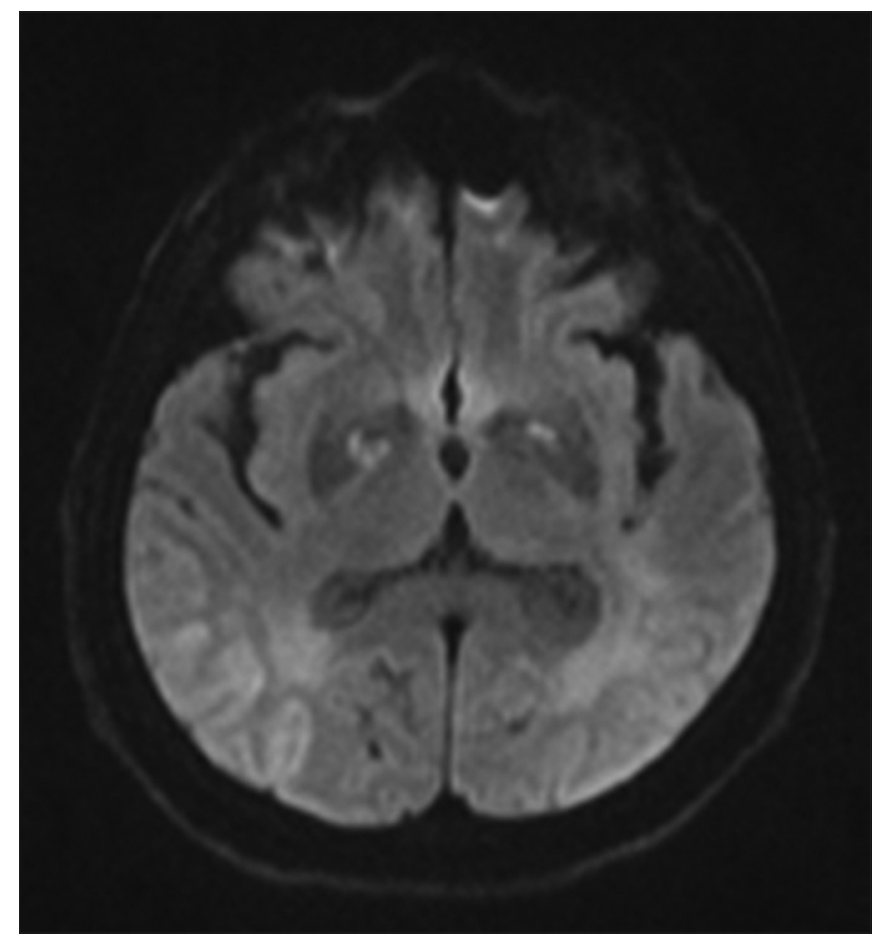

Fig. 6 A 65-year-old woman with hypoxic-ischaemic encephalopathy due to cardiac arrest. DW MR image shows restricted diffusion in both the globi pallidi and occipital cortices.

for the development of delayed neurological syndrome, as MR findings of delayed white matter changes correlate closely with the clinical manifestations of delayed neurological syndrome. ${ }^{(10-12)}$

Other than $\mathrm{CO}$ poisoning, many other conditions can give rise to hypoattenuating basal ganglia on $\mathrm{CT}$, with associated increased signal on T2-weighted or DW MR images. ${ }^{(7)}$ These include methanol and cyanide poisoning, hypoxia, hypoglycaemia, hyperammonaemia, Japanese encephalitis, sporadic Creutzfeldt-Jakob disease and metabolic conditions such as Leigh's disease and Wilson's disease. These conditions, however, show different patterns of involvement of the basal ganglia, thalami and cerebral cortex. Fig. 6 demonstrates the imaging findings of hypoxic-ischaemic encephalopathy secondary to cardiac arrest. It shows increased signal in both the globi pallidi on DW sequences, which is very similar to that seen in CO poisoning. Focal involvement of both the occipital cortices is, however, unusual for $\mathrm{CO}$ poisoning, thereby pointing to an alternative diagnosis in this case. Fig. 7 shows asymmetric involvement of the left lentiform nucleus in a patient with acute stroke, which differs from the typical symmetrical involvement of both the globi pallidi in $\mathrm{CO}$ poisoning. Furthermore, patients with conditions that may mimic CO poisoning on imaging studies generally have different clinical presentations and laboratory findings. As such, correlation of imaging findings with clinical history and laboratory data is of utmost importance when $\mathrm{CO}$ poisoning is suspected on imaging studies. ${ }^{(7)}$

Treatment of patients with $\mathrm{CO}$ poisoning is primarily supportive, with administration of $100 \%$ oxygen. Although some studies claim that hyperbaric oxygen therapy (HBOT) results in a reduction in delayed neurologic sequelae, cerebral oedema and pathologic central nervous system changes in patients with

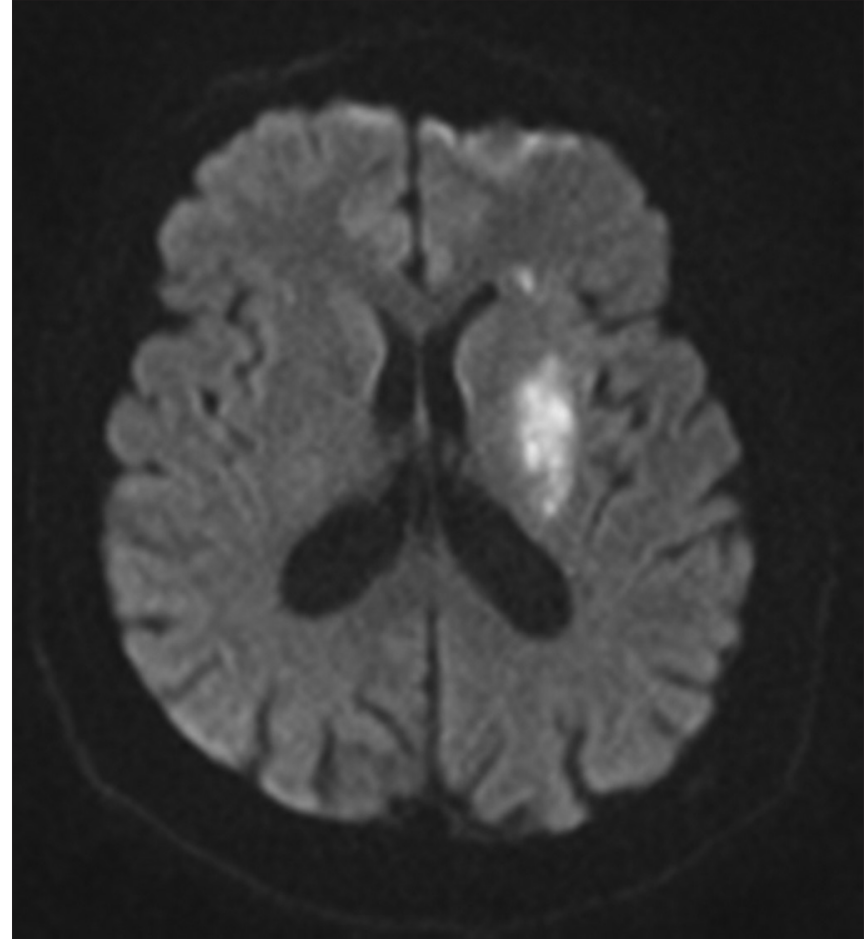

Fig. 7 A 74-year-old woman with acute stroke. DW MR image shows asymmetrical restricted diffusion in the left lentiform nucleus.

$\mathrm{CO}$ poisoning, universal treatment guidelines and criteria for treatment of $\mathrm{CO}$ poisoning patients with HBOT have not been established, as systematic reviews have not demonstrated a clear benefit of HBOT. ${ }^{(13,14)}$

In conclusion, although necrosis of the globus pallidus is the most common manifestation of acute $\mathrm{CO}$ poisoning on neuroimaging, other patterns of brain injury have been described. Abnormal appearances of the basal ganglia can also be caused by numerous other clinical and pathological conditions. Therefore, correlation of typical imaging features with clinical history and laboratory data is of utmost importance in arriving at the correct diagnosis.

ABSTRACT A 59-year-old man with a history of major depression was found by his wife to be unconscious and foaming at the mouth. On arrival at the emergency department, the patient was noted to be unresponsive. Computed tomography of the brain showed symmetrical ill-defined areas of hypoattenuation involving the medial aspects of both lentiform nuclei, while magnetic resonance images of the brain showed symmetrical increased signal in the bilateral globi pallidi on diffusion weighted, T2-weighted and fluid attenuated inversion recovery sequences. These findings were those of acute carbon monoxide poisoning. Despite aggressive treatment, the patient's condition continued to deteriorate and he eventually passed away. The various imaging findings of carbon monoxide poisoning in the brain and the differential diagnoses are discussed.

Keywords: basal ganglia lesions, carbon monoxide poisoning, globus pallidus necrosis, intracerebral lesions 


\section{REFERENCES}

1. Omaye ST. Metabolic modulation of carbon monoxide toxicity. Toxicology 2002; 180:139-50.

2. Thom SR, Bhopale VM, Fisher D. Hyperbaric oxygen reduces delayed immune-mediated neuropathology in experimental carbon monoxide toxicity. Toxicol Appl Pharmacol 2006; 213:152-9.

3. Gorman D, Drewry A, Huang YL, Sames C. The clinical toxicology of carbon monoxide. Toxicology 2003; 187:25-38.

4. Touger M, Gallagher EJ, Tyrell J. Relationship between venous and arterial carboxyhemoglobin levels in patients with suspected carbon monoxide poisoning. Ann Emerg Med 1995; 25:481-3.

5. Raub JA, Mathieu-Nolf M, Hampson NB, Thom SR. Carbon monoxide poisoning--a public health perspective. Toxicology 2000; 145:1-14.

6. Hampson NB, Hauff NM. Carboxyhemoglobin levels in carbon monoxide poisoning: do they correlate with the clinical picture? Am J Emerg Med 2008; 26:665-9.

7. Hegde AN, Mohan S, Lath N, Lim CC. Differential diagnosis for bilateral abnormalities of the basal ganglia and thalamus. Radiographics 2011; 31:5-30.

8. Lo CP, Chen SY, Lee KW, et al. Brain injury after acute carbon monoxide poisoning: early and late complications. AJR Am J Roentgenol 2007; 189:W205-11.

9. O'Donnell P, Buxton PJ, Pitkin A, Jarvis LJ. The magnetic resonance imaging appearances of the brain in acute carbon monoxide poisoning. Clin Radiol 2000; 55:273-80.

10. Chang KH, Han MH, Kim HS, Wie BA, Han MC. Delayed encephalopathy after carbon monoxide intoxication: MR imaging features and distribution of cerebral white matter lesions. Radiology 1992; 184:117-22.

11. Murata $T$, Kimura $H$, Kado $H$, et al. Neuronal damage in the interval form of $\mathrm{CO}$ poisoning determined by serial diffusion weighted magnetic resonance imaging plus $1 \mathrm{H}$-magnetic resonance spectroscopy. J Neurol Neurosurg Psychiatry 2001; 71:250-3.

12. Kim HJ, Chang KH, Song IC, et al. Delayed encephalopathy of acute carbon monoxide intoxication: diffusivity of cerebral white matter lesions. AJNR Am J Neuroradiol 2003; 24:1592-7.

13. Buckley NA, Isbister GK, Stokes B, Juurlink DN. Hyperbaric oxygen for carbon monoxide poisoning: a systematic review and critical analysis of the evidence. Toxicol Rev 2005; 24:75-92.

14. Buckley NA, Juurlink DN, Isbister G, Bennett MH, Lavonas EJ. Hyperbaric oxygen for carbon monoxide poisoning. Cochrane Database Syst Rev 2011; CD002041. 


\section{SINGAPORE MEDICAL COUNCIL CATEGORY 3B CME PROGRAMME} (Code SMJ 201408B)

Question 1. Regarding the clinical presentation of carbon monoxide (CO) poisoning:

(a) Patients may present with mild symptoms such as headache and malaise.

(b) Patients may present with deafness or blindness.

(c) Loss of consciousness is usually prolonged in moderate intoxication.

(d) Delayed neurological syndrome does not occur in patients who recover well from the acute symptoms of $\mathrm{CO}$ poisoning.

Question 2. Regarding neuroimaging of patients with CO poisoning:

(a) The caudate heads are most commonly involved.

(b) Computed tomography (CT) of the brain is superior to magnetic resonance (MR) imaging of the brain for evaluation of the basal ganglia in the acute setting.

(c) Necrotic globi pallidi may demonstrate contrast enhancement on MR imaging.

(d) Focal cortical injury has not been described in $\mathrm{CO}$ poisoning.

Question 3. Regarding the patterns of brain injury in CO poisoning:

(a) Diffuse hypoxic-ischaemic encephalopathy commonly occurs in patients with mild to moderate intoxication.

(b) Diffuse brain atrophy occurs early in the course of $\mathrm{CO}$ poisoning.

(c) Cerebral white matter demyelination is a late manifestation of $\mathrm{CO}$ poisoning.

(d) Cerebral white matter demyelination may be responsible for symptoms such as mental retardation and emotional disorders.

Question 4. Regarding the pathophysiology of CO poisoning:

(a) $\mathrm{CO}$ binds to haemoglobin up to 50 times more avidly than oxygen.

(b) $\mathrm{CO}$ can directly impair the function of respiratory cytochromes.

(c) $\mathrm{CO}$ can cause adverse effects resulting from leucocyte-mediated changes in the brain.

(d) The basal ganglia are more prone to hypoxic injury, as they have a high metabolic activity.

Question 5. Regarding laboratory testing and clinical management of patients with CO poisoning:

(a) Carboxyhaemoglobin levels can be accurately measured from venous samples.

(b) The carboxyhaemoglobin level in smokers is up to 30 times that in nonsmokers.

(c) Hyperbaric oxygen therapy has been definitively shown to improve the outcome of patients.

(d) Carboxyhaemoglobin levels do not accurately predict the clinical presentation or outcome of patients.

True False

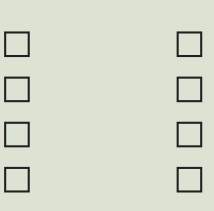

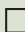

$\square$

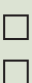

$\square$

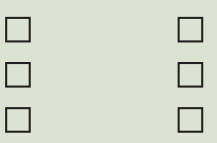

$\square$
$\square$
$\square$
$\square$
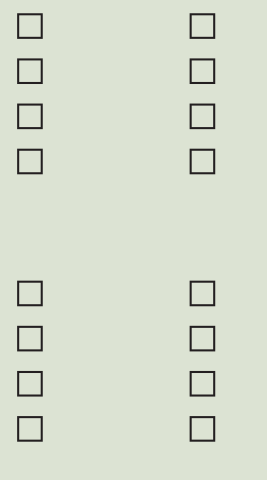

\section{Doctor's particulars:}

Name in full

MCR number

Specialty:

Email address

\section{SUBMISSION INSTRUCTIONS:}

(1) Log on at the SMJ website: http://www.sma.org.sg/publications/smjcurrentissue.aspx and select the appropriate set of questions. (2) Provide your name, email address and MCR number. (3) Select your answers and click "Submit".

\section{RESULTS:}

(1) Answers will be published in the SMJ October 2014 issue. (2) The MCR numbers of successful candidates will be posted online at the SMJ website by 30 September 2014. (3) Passing mark is $60 \%$. No mark will be deducted for incorrect answers. (4) The SMJ editorial office will submit the list of successful candidates to the Singapore Medical Council. (5) One CME point is awarded for successful candidates.

Deadline for submission: (August 2014 SMJ 3B CME programme): 12 noon, 24 September 2014. 